\title{
SETS AND SUBSERIES
}

\author{
G. M. PETERSEN
}

Suppose $\alpha_{n} \neq 0$ for all $n$. By replacing a set of terms from the series $\sum \alpha_{n}$ by 0 's, we form a subseries of the original series. These subseries can be put in one to one correspondence with the non-terminating binary expansion of the points on the real line segment $(0,1)$. The point $\xi=. b_{1} b_{2} \ldots b_{n} \ldots$ shall correspond to a subseries if and only if $b_{n}=0$ whenever the $n$th term of the original series has been replaced by 0 and $b_{n}=1$ whenever the $n$th term is retained. We can now speak of sets of subseries of the first category, measure zero, etc.

If we have a series $\sum a_{n}$ whose terms $\left\{a_{n}\right\}$ form a positive monotone decreasing sequence, $a_{1} \geqslant a_{2} \geqslant \ldots \geqslant a_{n} \geqslant \ldots$, then it is easy to show by a Dedekind section the existence of an index $p$ such that $\sum a_{n}{ }^{q}$ converges for $q>p$ and diverges for $q<p$. The object of this note is to show that if the terms of a series are a positive monotone decreasing sequence, the set of subseries that have an index differing from the original series is of the first category.

We first prove a lemma.

Lemma. If $\left\{c_{n}\right\}$ is a positive monotone decreasing sequence, $\sum c_{n}$ diverges, $\lim \sup n c_{n} \neq 0$, then the set of subseries that converges is of the first category.

Proof. We can assume that $2 n(m) c_{2 n(m)}>k$ where $\{n(m)\}$ is a subsequence of $\{n\}$. However, this implies $n(m) c_{n(m)}>\frac{1}{2} k$ and $\nu c_{\nu}>\frac{1}{2} k$ for $n(m) \leqslant \nu \leqslant 2 n(m)$. We can associate with this sequence a regular summation method $A$, defined by

$$
t_{m}=\frac{1}{n(m)} \sum_{\nu=n(m)}^{n(m)} s_{\nu}
$$

Suppose now that $c^{\prime}{ }_{n}=c_{n}$ if the term is retained for the subseries and $c^{\prime}{ }_{n}=0$ otherwise. For any subseries $\sum c_{n}^{\prime}$ we shall choose a sequence of 0 's and 1's, $\left\{s_{n}^{\prime}\right\}$, so that $s_{n}^{\prime}=1$ if $c_{n}^{\prime} \neq 0$ and $s_{n}^{\prime}=0$ otherwise. There is an evident correspondence between the sequence $\left\{s_{n}^{\prime}{ }_{n}\right\}$ and the point corresponding to the subseries. We now see that if $\sum c_{n}^{\prime}$ converges, $\left\{s_{n}^{\prime}\right\}$ must be $A$ summable to 0 . For if $t_{m_{\mu}} \geqslant \lambda>0$ for an infinite set $\{\mu\}$, then

$$
\sum_{\nu=n\left(m_{\mu}\right)}^{2 n\left(m_{\mu}\right)} c^{\prime}{ }_{\nu}>\lambda n\left(m_{\mu}\right) c_{2 n\left(m_{\mu}\right)}>\lambda \frac{k}{2}
$$

and the series diverges.

Received June 15, 1956. 
However, it has been shown by Hill that the set of points on $(0,1)$ in the binary expansion corresponding to such a set of 0 's and 1's is of the first category.

We are now ready to prove our theorem.

THEOREM. If $\left\{a_{n}\right\}$ is a positive monotone decreasing sequence, the set of subseries of $\sum a_{n}$ with a different index is of the first category.

Proof. The index for the series $\sum a_{n}$ may be assumed to be 1 . For if the index is $p, \sum{a_{n}}^{p}$ will have an index 1 and the set with a different index will be the same for $\sum a_{n}$ and $\sum a_{n}{ }^{p}$.

If $r<1$, then $\lim \sup n a_{n}{ }^{r}>0$, for if

$$
\lim _{n \rightarrow \infty} n a_{n}^{r}=0,
$$

then $a_{n}=0\left(n^{-1 / r}\right)$ and for $r<q<1, \sum a_{n}{ }^{q}$ would converge, contrary to our assumption. Hence the set of subseries of $\sum a_{n}{ }^{r}$ that converges for any $r$ is of the first category.

If a subseries has an index less than 1 , then it will belong to the set $E_{\nu}$ of convergent subseries of

$$
\sum a_{n}^{1-1 / \nu}, \quad \nu=2,3, \ldots
$$

for some $\nu$. The set of all subseries with index less than 1 will be contained in the union of these sets and so will be of the first category.

\section{REFERENCE}

1. J. D. Hill, Summability of sequences of 0's and 1's, Annals of Math., 46 (1945), 556-62.

University College of Swansea 